\title{
PANEL ON FUTURE CHALLENGES IN MODELING METHODOLOGY
}

\author{
Simon J. E. Taylor (Convener) \\ Centre for Applied Simulation Modeling \\ Department of Information Systems and Computing \\ Brunel University \\ Uxbridge, Middx UB8 3PH, U.K.
}

\section{Ray J. Paul}

\author{
Centre for Applied Simulation Modeling \\ Department of Information Systems and Computing \\ Brunel University \\ Uxbridge, Middx UB8 3PH, U.K.
}

\author{
Steffen Straßburger \\ Fraunhofer Institute for Factory Operation \\ and Automation \\ Department Virtual Development \\ Sandtorstraße 22, 39106 Magdeburg, GERMANY
}

\author{
Peter Lendermann \\ Production and Logistics Planning Group \\ Singapore Institute of Manufacturing Technology \\ 71 Nanyang Drive, Singapore 638075, SINGAPORE
}

\author{
Steven W. Reichenthal \\ Boeing \\ 3370 Miraloma Ave. \\ Anaheim, CA 92803, U.S.A.
}

\begin{abstract}
This panel paper presents the views of six researchers and practitioners of simulation modeling. Collectively we attempt to address a range of key future challenges to modeling methodology. It is hoped that the views of this paper, and the presentations made by the panelists at the 2004 Winter Simulation Conference will raise awareness and stimulate further discussion on the future of modeling methodology in areas such as modeling problems in business applications, human factors and geographically dispersed networks; rapid model development and maintenance; legacy modeling approaches; markup languages; virtual interactive process design and simulation; standards; and Grid computing.
\end{abstract}

\section{INTRODUCTION}

Each year at the Winter Simulation Conference there are panels that convene to discuss various fascinating, forward-looking topics. In 2003 these included (in no particular order) the future of simulation, the future of simulation technology, distributed simulation, education and the ROI of simulation. All of these have some impact on modeling methodology. To contribute to this set of annual stimulat- ing discussions, and to consider these from the perspective of modeling methodology, this panel has been convened. Each of the panelists was given the task of considering what to them are the future challenges in modeling methodology. These include modeling problems in business applications, human factors and geographically dispersed networks; rapid model development and maintenance; legacy modeling approaches; markup languages; virtual interactive process design and simulation; standards; and Grid computing. It is hoped that this will provide a wide perspective on the future of modeling methodology.

\section{POSITION STATEMENT OF PETER LENDERMANN}

This contribution is specifically looking from the point of view of discrete event simulation as a tool for virtual experimentation to enable design and performance enhancement in manufacturing and logistics. In a world of increasing complexity and customization it will be very important to make sure that the complexity of the systems that simulations are supposed to represent does not develop faster than the capability to model these systems. To achieve this, modeling techniques will have to meet five major requirements as described in the following sections. 


\subsection{Representation of Business Applications}

Firstly, modeling techniques have to take into account the specific characteristics of today's pull-environments, in which operational execution plans are the result of a translation from frequently-changing customer demand into material quantities to be released into and moved within the manufacturing and logistics systems at pre-specified times. These complex operational decisions are increasingly taken over by scheduling systems. Therefore, high-fidelity models can be created only if these scheduling systems are incorporated into the simulation. Researchers at the Singapore Institute of Manufacturing Technology (SIMTech) have previously highlighted the importance of this issue and described relevant research achievements at the Winter Simulation Conference (see e.g. Lendermann 2003).

\subsection{Representation of Human Factors}

Secondly, in complex manufacturing and logistics environments there will always remain some operational decisions that are taken by humans. Therefore it will be necessary to be able to represent, model and analyze human intelligence and behavior, and sometimes even effects such as fatigue. On the application level, agent-based simulation could play a more important role in the future to achieve this.

\subsection{Representation of Geographically Dispersed Networks}

Thirdly, in today's complex supply networks, events often depend on what is happening at geographically distant locations. This is where distributed simulation technology comes into the picture. An example of how distributed simulation can be applied to study complex scenarios to enhance the performance of a semiconductor supply chain is given in (Chong 2004).

\subsection{Rapid Model Development}

Also, today's manufacturing and logistics systems have to be designed fast: Many initiatives such as $\mathrm{MDA}^{\mathrm{TM}}$ (<www. omg.org/docs/omg/03-06-01.pdf>), RMODP (<www.dstc.edu.au/Research/Projects/ ODP/ref_model.html>), DEVS (<www.sce.carl ton.ca/faculty/wainer/standard/devs-tor. pdf>), or OASIS (<www.oasis-open.org / home/ index.php >) have been pursued to develop the required information technology standards. Other initiatives such as the HLA-CSPIF (<www.cspif.com>), a worldwide forum consisting of users, vendors and researchers to integrate and enable interoperability between commercial off-theshelf (COTS) simulation packages are under way. However, a really significant reduction of the cycle time for simulation modeling will only be possible if standardization also takes place on the application level that would result in archived, re-usable simulation model components that require much less customization effort.

\subsection{Flexible Model Maintenance}

Lastly, once manufacturing and logistics systems are designed they will continuously change throughout their lifetime. Therefore it is necessary to be able to catch up with simulation model maintenance in as a flexible manner as possible. This could be enabled through symbiotic systems that interact with the business application in a mutually beneficial way and has been classified as a Grand Challenge by the research community (see Dagstuhl 2003): A symbiotic system is highly adaptive in a sense that the simulation system not only performs what-if experiments that are used to enhance the physical system, but also accepts and responds to data from the physical system. The physical system benefits from the optimized performance that is obtained from the analysis of simulation experiments. In turn, the simulation system benefits from the continuous supply of the latest input data and the automatic validation of its outputs.

\section{POSITION STATEMENT OF RAY J PAUL}

My position is the "Problem Solving Minus the Simulation Diversionary Triathlon." A future challenge in modeling methodology is to rid ourselves of the major limiting factor in problem solving - legacy thinking! Problem solving will be improved by disavowing ourselves of these diversionary modeling activities. This position selects three from this Triathlon for attention.

- Model accuracy as exemplified by VV\&A. The problem to be solved becomes forgotten in the pursuit of modeling accuracy. Model accuracy diverts thinking from problem solving activities to modeling activities. The problem becomes a victim of model - the model is highly accurate but solves the wrong problem. This point is lost by those who have created the model as they are convinced that the accuracy of the model will solve the problem that is now a distant memory.

- Statistical output analysis. There is a vast literature that feeds itself and there is little evidence of practical use. Anecdotally, the overwhelming response to "what I did with my simulation" is "I ran it 10/20/100 times and calculated the mean of the response." Theory and practice might eventually come together if it was not for the observation that the theory is intrinsically wrong. For example, output streams from simulation models are not independently identically distributed (IID). The only way to make them so is to throw away most of the output. Also, models are constructed with great intellectual effort to capture the interactions in a sys- 
tem. All this knowledge is cheerfully thrown away, ignored, or wasted in the analysis of the output.

- Bigger is better. Bigger models better represent the real world. Who needs this? The real world is complex and misunderstood. Modeling attempts to simplify this for understanding. A big model therefore adds to the complexity and misunderstanding of the real world and extra misunderstanding in the model itself. The net gain is twice the pain! Single models well understood might explain something about the complex real world. Any model that is not understood is helping us keep problem understanding at bay. A possible approach to this is grab and glue, a quick and dirty approach that emphasizes appropriate input/output mechanisms so that the right problem is solved and not the right model (Paul and Taylor 2002).

I hope the examples of the Triathlon challenge contemporary views on "legacy modeling." I again remind those who have forgotten, and introduce those who have not heard, the words of the great Kiviat who at the 1990 Winter Simulation Conference brought to us the concept of SINSFIT - Simulation is No Substitute for Intelligent Thinking. I urge readers of this panel paper to again go forth and think!

\section{POSITION STATEMENT OF STEVE REICHENTHAL}

My position regarding future challenges in modeling and simulation pertains to markup languages developed for simulation modeling. While this is still a relatively open frontier for the simulation community, lessons may be learned by observing the evolutions of HTML, web browsers, and XML.

Markup, as we know it today, was created in the 1970's with SGML and was used in the narrow field of high-end electronic publishing for about 20 years until the creation of the web and HTML brought it into a broader audience. Yet, even today, end users generally don't care much about HTML, because all browsers support a single standard - imagine if that was not the case. Nevertheless, web developers today do care about HTML, but don't try to build their own web browsers. It took several years, however, for web browsers to become generally useful in application development, and during that time the earlier ways of providing user interfaces dominated:

- They were created from scratch

- Their basic features were well understood but loosely integrated

- They were hard-coded to their applications

- Their development required a high degree of programming skill and high cost

- Their building blocks were significantly different when using one vendor's tool vs. another
We can say the same things about developing simulations today, but no longer about user interfaces. Early HTML was weak in that it could only provide text, graphics, and hyperlinks, so several significant features were added over time which eliminated its shortcomings: behavior through scripts, plug-ins, the Document Object Model, etc. Now, functionality scripted in HTML is balanced with that provided by the web browser and plug-ins.

Leveraging the popularity of HTML, XML arrived several years later as an easier-than-SGML way to create an HTML-like markup language. The XML phenomenon has spawned the creation of many markup languages and particularly in the area processes modeling, where several have emerged. What would have happened if XML was popularized before HTML? Could it have been made popular without HTML? Would there have been several HTMLs? These are rhetorical questions. However, I believe that the future technical challenges for any useful simulation modeling language would be to provide a similar utility as that afforded by HTML, with its added scripting, plug-ins, and a general purpose object model. In an effort to understand the challenges surrounding such a language, The Simulation Reference Markup Language (SRML) was developed to have HTML-like capabilities specifically targeted for simulation model interchange, and a study is underway at the Simulation Interoperability Standards Organization (SISO) to evaluate the standardization issues for such a language.

Communities of interest have formed at standards organizations which promote interoperability and interchange through a consensus-based approach. One promising XML modeling standard, which is currently under definition at SISO, is called Encapsulated Base Object Models (ECAP-BOMs). The basic goal of BOMs is to facilitate modeling and simulation interoperability, reuse, and composability through XML. Once developed, BOMs would offer a standard way to define the communication patterns which would be required among components, as well as to provide a means for encapsulating packages of data and behavior (objects) that could be shared among diverse simulation tools - hence Encapsulated BOMs. The technical challenges facing ECAP-BOMs are not difficult, given that basic interchange problems have been solved by the groups building web browsers, web servers, and other applicable web technologies, like SRML. However BOMs can only go so far in that an interest group will need to be established in order to standardize the specific communication patterns needed for process-oriented BOMs (P-BOMs) to interact. Encapsulated P-BOMs could potentially be a useful standard that would enable various simulation packages to interchange models. A more difficult challenge facing BOM standards in the near term will be that of gaining broad support of an interested set of developers and vendors which could influence and support future BOM development and use. 


\section{POSITION STATEMENT OF STEFFEN STRAßBURGER}

My position is based on the challenge of VirtualInteractive Process Design and Simulation. Looking at the advances in simulation methodology in the past years many scientists share the author's opinion that no major breakthroughs have been invented in the past years (Barton et al. 2003). One exception could be seen in the development of the HLA standard which for the first time provides a standardized architecture for simulation interoperability which is in principle applicable in the entire simulation community. Although far from being applied widely in industry, HLA is certainly a success in an area where previous attempts have failed (Straßburger 2001).

Although simulation methodology apparently has not changed so much in the past years, many advances have been made in the area of simulation tools (so-called commercial off-the-shelf simulation software). On the one hand these advances promise easier simulation model creation. The extent of fulfillment of this promise is another issue, which is not discussed here, see (Rehn 2004) for some interesting insights on this issue. On the other hand much effort has been put into the area of simulation output visualization.

For simulation systems with no built-in graphics capabilities, the animation system Proof Animation (Henriksen 2000) has been a reliable partner over the years for $2 \mathrm{D}$ visualization. It offers post-processed and concurrent animation capabilities on the Windows platform. For many simulation problems 2D-visualisation is still the most efficient visualization method considering the effort needed to build the animation and the benefit gained from it.

Increasingly more simulation systems tend to offer different variants of 3D-animation. Some tools offer 3Dcapabilities on-top of the normal simulation model generation (e.g. Automod, eM-Plant), in other tools the modeling process is directly done in a 3D environment (e.g. QUEST). The general trend in industry towards the requirement of 3D visualization is connected to the rise of the vision of the Digital Factory, heavily promoted by the automotive industry.

The next logical step in this development is the introduction of immersive and interactive environments based on Virtual Reality (VR) techniques. In such environments users can experience their simulation model, they can walk through their virtual factories, they can visually inspect the different stations, analyze bottlenecks, etc.

Most importantly these environments can provide interactivity in different ways.

- The user can obtain additional information by interacting with the visualization component, e.g., by selecting a station and requesting a detailed statistics about its usage.
- The user can interactively modify the simulation run by changing routings, processing times, worker allocations, etc. Thus the user can experiment with the model in an immersive environment as if he was standing in a real factory.

- The user can interactively modify the simulation model. This case is partially similar to the previous alternative, with the difference that the users actions indeed change the simulation model of the simulator permanently.

- $\quad$ The user can be inserted into the simulation, e.g., take over tasks of workers which are normally part of the simulation. This can be done for training purposes, e.g., to show workers which effects certain actions will have.

The technical basis for enabling such virtual environments is typically achieved by coupling one or more simulation systems with a virtual reality system. This can be based on standards like HLA or simple network interfaces like TCP/IP. Using standards like HLA has the advantage that issues like synchronization between the VR and the simulation are automatically taken care of. In pilot projects at the Fraunhofer IFF different simulators and VR environments have been connected.

The overall objective of the described concept is to establish VR as a command and control tool for factory design and factory operation. The virtual world could act as the integration platform for different simulation models (e.g. OEM supplier models developed in different simulation tools). In the design phase of the factory, the processes of the planned factory could be tested and optimized within the VR world.

In the operation phase of the factory, the VR world could act as the virtual representation of the real factory. Based on on-line simulation concepts (Schulze et al. 1999) and a connection to the shop-floor systems, the state of the VR world (and the connected simulations) would reflect the state of the real factory. In case of emergencies (e.g. machine failure) the factory operator could plan and test different plans of action (fast-forward simulation) and select the best option. Even for daily work planning the virtual factory could provide the optimal basis since it is initialized with the right initial conditions for all work stations and it could have the connection to the relevant ERP (enterprise resource planning) and order systems.

In summary, the topic of virtual-interactive process design and simulation in immersive environments can be regarded the next logical step in the development of visual 3D simulation and is a logical consequence derived from the requirements of the Digital Factory. The vision of VR based integration platforms is supported by technologies like HLA, which help to integrate different simulation systems. 


\section{POSITION STATEMENT OF SIMON J E TAYLOR}

In our discussion of "Future Challenges in Modeling Methodology" I would like to consider whether or not standards have any worth in the domain of simulation modeling represented by users of the range of so-called COTS Simulation Packages (CSPs) used to analyze problems in dynamic, discrete event-based systems (e.g. Arena, Witness, Simul8, etc.) The CSPs are used to aid simulation modeling tasks such as model building, experimentation, visualization, and reporting in a wide range of areas including commerce, health and manufacturing. A CSP is therefore technology used by a simulation modeller to solve a problem. However, it also influences the concepts and methods that the simulation modeller is able to bring to bear on a problem.

Arguably, there are two current standards in simulation modeling: the IEEE 1278 standard Distributed Interactive Simulation (DIS) and the IEEE 1516 High Level Architecture (HLA). The first of these is inappropriate for CSPbased simulation modeling as it deals with various domainspecific aspects of warfare simulation in a distributed environment. The second also concerns simulation in a distributed environment but is domain-neutral (i.e. any information to be exchanged between simulations (federates) can be specified). In support of the type of simulation modeling described above, only the HLA standard is relevant - and then only to the execution of CSPs and their models over a networked environment. Issues concerning the use of the HLA to support CSP interoperability have been the subject of a previous panel at the Winter Simulation Conference (Taylor et al. 2003). The Simulation Interoperability Standards Organization (SISO) seeks to further the "cause" of interoperability standards through its Standards Activity Committee (SAC). In SISO terms, new standards are termed "products" and it is the role of Product Development Groups (PDGs) to develop new products. When a product matures it is balloted, i.e. the community in which the product sits votes on whether or not the product is appropriate. In this respect SISO and its practices are similar to the body responsible for standards development in the World Wide Web (recommendations and the W3C). For example, the CSP Interoperability Forum (<www . cspif . com>) has existed as an international body since August 2002. This forum is dedicated to creating standard approaches to CSP interoperability. SISO oversees the development of interoperability standards. If the CSPIF is to have "credibility" for their work, the natural choice is for the CSPIF to become a PDG as SISO is a recognized body linked to an IEEE standard.

From the above, there is a clear "home" for simulation interoperability standards and that one facet of emerging CSP use is well supported by that organization. However, interoperability standards arise from a requirement of distributed computing and not from simulation modeling. Do formal, organizationally "accredited" standards therefore have a significant role to play in the life of the practitioner who uses a CSP to develop a single "standalone" models?

As indicated above, a CSP is not just a visual interactive tool - it also strongly influences the concepts and methods that the simulation modeller brings to bear on a problem. For example, each CSP is typically based on some variant of the discrete event simulation paradigm. Models change state at discrete points in time by scheduled or conditional events and typically represent entities or objects (documents, patients, parts, trains, etc.) in some form that pass through networks of queues and workstations (work queuing at a desk in an office, patients waiting to see a doctor, parts buffered for machining, trains waiting at a station, etc.) Each CSP essentially does what its competitor CSPs do. However, each does its own tasks in its own way. Consider the entity. Across the range of CSPs, an entity is identifiable as an entity, an element, an item, an object, a transaction, etc. Artefacts such as queues and workstations are recognizable between CSPs but differ in name and slightly in functionality. Further, the simulation engine differs between CSPs, thereby enforcing different behavioural rules between models. Overall, there are few standard conventions across packages. This has led to a polarization of the CSP practitioner community starting in education at degree-level courses at University and on in to industry.

Does this matter? The current status quo appears to be that practitioners are happy with their lot but subdivided into user-based communities. Models developed in different packages cannot be interchanged and occasionally this can be a problem when larger organizations have not "standardized" on which CSP should be used throughout the organization. However, this will be further exacerbated with increasing attempts to use simulation modeling to study larger inter-organization problems such as supply chains. Additionally, expensive skills developed based around one CSP can be transferred to another but at a cost. Surely it would be a beneficial for practitioners to have a "universal" set of "standard" simulation approaches that are "mapped" onto a CSP?

While this is important, the real problem is this. In the simulation interoperability community there is a standard "language" based around the HLA that allows large community discussions (such as those at the Simulation Interoperability Workshops) over a wide range of tool, conceptual and methodological issues. This critical mass has allowed that community to make substantial advances in a relatively short period of time. In the CSP world, separate, polarized CSP-based communities exist. Methodological and technological advances are determined by the evolution of the CSP and therefore by the vendor of that CSP. Would the pace of change of methodology and technology be better led by the community and not the software vendor, much as the World Wide Web has developed under the auspices of the $\mathrm{W} 3 \mathrm{C}$ ?

If this is true, would a formal, organizationally "accredited" standard concerning a range of issues from a formal 
definition of "entity" to a common simulation project lifecycle therefore have a significant impact on simulation practitioners? If the achievements of those involved in the simulation interoperability community is evidence of what can happen if a common "standard" ground is achievable, then I believe that if practitioners, vendors and academics of the various CSP communities can pull together to produce such a standard, under an existing or new organization, then the critical mass engendered by this new commonality could usher in a new era in simulation modeling and be a worthy future challenge in modeling methodology.

\section{POSITION STATEMENT OF STEPHEN J TURNER}

My position on the future of modeling methodology is taken from the viewpoint of large scale distributed simulation on the Grid. Simulation plays an important role in many areas of industrial production, business, education, engineering and science. It is a powerful tool for investigating and evaluating complex scenarios such as predicting the behavior of new industrial systems or for analyzing the effects of adverse weather conditions on air traffic. Nowadays, the development of complex simulation applications usually requires collaborative effort from analysts with different domain knowledge and expertise, possibly at different locations. Furthermore, these simulation systems often require huge computing resources and the data sets required by the simulation may also be geographically distributed. In order to support collaborative model development and to cater for the increasing complexity of such systems, it is necessary to harness distributed resources over the Internet.

In recent years, there has been an explosion of interest in large scale distributed simulation. Much of this activity has centered around the High Level Architecture (HLA) for simulation (Dahmann et al. 1998), an IEEE standard to facilitate interoperability among simulations and promote reuse of simulation models. Using HLA, a large-scale distributed simulation can be constructed by linking together a number of geographically distributed simulation models (or federates) into an overall simulation (or federation). However, the HLA does not provide any support for collaborative development of simulation applications, neither does it provide any mechanism for managing the resources where the simulation is being executed.

Grid technology (Foster 2004a) enables collaboration and the use of distributed computing resources, while also facilitating access to geographically distributed data sets. Our vision is a "Grid plug-and-play distributed simulation system", a collaborative distributed simulation environment where analysts at different locations develop, modify, assemble and execute distributed simulations over the Grid (Theodoropoulos et al. 2003). However, a number of important new research challenges need to be addressed before this vision is realized. First, a basic infrastructure providing services to support model discovery and composition is es- sential in the development of collaborative distributed simulations. Secondly, to conduct simulation experiments easily over distributed resources from different organizations, mechanisms that can provide coordinated and secured simulation executions are required. In addition, to meet the performance requirements demanded by large scale distributed simulations, resource management mechanisms that balance the load and provide fault-tolerance capabilities are needed.

A Grid computing environment consists of a collection of heterogeneous, dynamic, shared resources. These resources may be located at different geographical places and may belong to different administrative domains. The emergence of Grid services (Foster 2004b) and the potential for seamless aggregation, integration and interaction makes it possible to combine computations, experiments, observations, and data to form a powerful simulation environment. Zong et al. (2004) describes a framework for executing HLA-based distributed simulations using Grid services. The RTI control process is managed by an RTI Service and can be dynamically discovered. Simulation models are encapsulated within Federate Services and are assembled through their Grid interface to form a large scale distributed simulation. As different models can be dynamically located, it provides great flexibility. Reusability is inherently provided by the nature of Grid services.

Service composition offers a new and evolving paradigm for building simulation applications. Suppose that semantic meta-data, expressed for example by means of ontologies, is associated with the models and accessed via a Grid index service. Then a user could locate a model or models that provided certain capabilities by searching the semantic meta-data of the models registered with the index service. Ideally, once a set of component models has been discovered, the semantic meta-data could be checked to determine if the models could be composed in a meaningful way. However, while there is much ongoing research into the interoperability and composability of simulation models (Weisel et al. 2004), achieving this kind of semantic composability is a challenging task.

To provide effective resource management, a number of research issues must be considered. These include resource discovery, federate deployment, load monitoring, dynamic load-balancing, check-pointing and fault-tolerance. We can make use of Grid services to perform the tasks of resource monitoring, coordination of simulation execution and security, while the RTI is used to perform simulation related tasks such as synchronization and time management. Cai et al. (2002) describes a prototype Load Management System (LMS) developed to support the execution of HLA-based simulations over geographically distributed computing resources. Using Grid services, the LMS will match-make the resource requirements of an HLA-based simulation and the resources managed by the resource sharing system, carry out authentication and authorization, schedule the simulation and provide mechanisms for load-balancing and faulttolerance during the simulation. 
Future directions of research include mechanisms to facilitate the discovery, composition and deployment of component simulation models using Grid services. Challenges remain to explore suitable formal approaches to the visual construction, validation and verification of composite simulation applications and to develop techniques (e.g., mobile agents) for automating the deployment and execution of such systems. New workflow languages are required that describe the various component models that constitute the simulation application together with the interactions between them.

\section{SUMMARY}

This panel paper has presented six different views on the future of modeling methodology. We hope that this will provoke a stimulating debate at this year's WSC and highlight priority research areas that will benefit our community at large.

\section{REFERENCES}

Barton, R., P. Fishwick, J.O. Henriksen, R.G. Sargent, and J.M. Twomey. 2003. Panel: Simulation - Past, Present and Future. In Proceedings of the 2003 Winter Simulation Conference, ed. S. Chick, P. J. Sánchez, D. Ferrin, and D. J. Morrice, 2044-2050. New York, N.Y.: Association for Computing Machinery Press. Available online via <http: //www . informs-Cs.org/wsc03papers/264.pdf > [accessed August 20, 2004].

BOM. 2003. Base Object Model (BOM) Template Specification Volume I - Interface BOM, SISO-STD-003.1DRAFT-V0.9

Cai, W., S.J. Turner, and H.F. Zhao. 2002. A Load Management Systsem for Running HLA-based Distributed Simulations over the Grid, Proc. 6th IEEE International Workshop on Distributed Simulation and Real Time Applications, 7-14. Piscataway. New Jersey: Institute of Electrical and Electronics Engineers.

Chong, C.S., P. Lendermann, B.P. Gan, B.M. Duarte, J.W. Fowler, and T.E. Callarman. 2004. Analysis of a Customer-Demand Driven Semiconductor Supply Chain in a Distributed Simulation Testbed. Accepted for the 2004 Winter Simulation Conference.

Dagstuhl. 2003. <http://www.informatik. uni-rostock. de/ lin/GC/report/

PADS . html > [accessed July 5, 2004].

Dahmann, D., F. Kuhl, and R. Weatherly. 1998. Standards for Simulation: As Simple As Possible But Not Simpler - The High Level Architecture for Simulation. Simulation 71(6): 378-387.

Foster I. and C. Kesselman. 2004a. Concepts and Architecture, The Grid 2: Blueprint for a New Computing In- frastructure. ed. I. Foster and C. Kesselman. 37-64. Morgan Kaufmann.

Foster I., C. Kesselman, and S. Tuecke. 2004b. The Open Grid Service Architecture, The Grid 2: Blueprint for a New Computing Infrastructure. ed. I. Foster and C. Kesselman. 215-258. Morgan Kaufmann.

Henriksen, J.O. 2000 Adding Animation to a Simulation Using Proof ${ }^{\mathrm{TM}}$. In Proceedings of the 2000 Winter Simulation Conference, ed. S. Andradóttir, J. A. Joines, R. R. Barton, K. Kang, and P. A. Fishwick, 191-196. New York, N.Y.: Association for Computing Machinery Press. Available online via <http://www. informs-Cs.org/wsc00papers/028.pdf > [accessed August 20, 2004].

Lendermann, P., N. Julka, L.P. Chan, and B.P. Gan. 2003. Integration of Discrete Event Simulation Models with Framework-Based Business Applications. In Proceedings of the 2003 Winter Simulation Conference, ed. S. Chick, P. J. Sánchez, D. Ferrin, and D. J. Morrice, 1797-1804. New York, N.Y.: Association for Computing Machinery Press. Available online via <http: //www. informs-cs.org/wsc03papers/230. pdf $>$ [accessed August 20, 2004].

Paul, R.J. and S.J.E. Taylor. 2002. What Use is Model Reuse: Is There a Crook at the End of the Rainbow? In Proceedings of the 2002 Winter Simulation Conference, ed. E. Yücesan, C.-H. Chen, J. L. Snowdon, and J. M. Charnes, 648-652. New York, N.Y.: Association for Computing Machinery Press. Available online via <http: //www. informs-cs.org/wsc02 papers / 083 .pdf > [accessed August 20, 2004].

Rehn, G. D. 2004. Future Trends in the Application and Execution of Simulation in US Industry. In Proceedings of the Conference "Simulation und Visualisierung 2004”, ed. T. Schulze, S. Schlechtweg, V. Hinz, SCS Europe Publishing House, Erlangen, San Diego, 1-14..

Schulze, T., S. Straßburger, and U. Klein. 1999. On-line Data Processing in Simulation Models: New Approaches and Possibilities through HLA. In Proceedings of the 2002 Winter Simulation Conference, ed. P. A. Farrington, H. B. Nembhard, D. T. Sturrock, and G. W. Evans, 1602-1609. New York, N.Y.: Association for Computing Machinery Press. Available online via <http: //www. informs-cs.org/wsc99 papers / 232 .pdf > [accessed August 20, 2004].

SRML. 2002. SRML - Simulation Reference Markup Language W3C Note, <http://www.w3.org/TR/ SRML/ > [accessed December 18, 2002].

Straßburger, S. 2001. Distributed Simulation Based on the High Level Architecture in Civilian Application Domains. Ghent: Society for Computer Simulation International, ISBN 1-56555-218-0.

Taylor, S.J.E., B.P. Gan, S. Straßburger, and A. Verbraeck. 2003. HLA-CSPIF Technical Panel on Distributed Simulation. In Proceedings of the 2003 Winter Simulation Conference, ed. S. Chick, P. J. Sánchez, D. Ferrin, 
and D. J. Morrice, 881-887. New York, N.Y.: Association for Computing Machinery Press. Available online via <http://www. informs-cs.org/wsc03 papers / 107.pdf > [accessed August 20, 2004].

Theodoropoulos, G., S.J. Turner, W. Cai, and B. Logan. 2003. Large Scale Distributed Simulation on the Grid, EPSRC e-Science Sister Project GR/S82862/01.

Weisel E.W., M.D. Petty, and R.R. Mielke. 2004. A Survey of Engineering Approaches to Composability. In Proceedings of the 2004 Spring Interoperability Workshop, 04S-SIW-105. Orlando, Florida: Simulation Interoperability Standards Organization.

Zong, W., Y. Wang, W. Cai and S.J. Turner. 2004. Grid Services and Service Discovery for HLA-based Distributed Simulation, submitted to 8th IEEE International Symposium on Distributed Simulation and Real Time Applications. Piscataway. New Jersey: Institute of Electrical and Electronics Engineers.

\section{AUTHOR BIOGRAPHIES}

PETER LENDERMANN is a Senior Scientist at the Singapore Institute of Manufacturing Technology (SIMTech) where he currently leads the D-SIMLAB Research Programme. He holds a concurrent appointment as Adjunct Senior Research Fellow at the Department of Industrial and Systems Engineering at the National University of Singapore. Previously he was a Managing Consultant with agiConsult in Germany where his focus was on the areas of supply chain management and production planning. He also worked as a Research Associate at the European Laboratory for Particle Physics CERN in Geneva (Switzerland) and Nagoya University (Japan). He obtained a Diploma in Physics from the University of Munich (Germany), a Doctorate in Applied Physics from HumboldtUniversity in Berlin (Germany) and a Master in International Economics and Management from BocconiUniversity in Milan (Italy). His research interests include modeling and analysis of manufacturing and logistics systems as well as distributed simulation. His email address is <peterl@SIMTech.a-star.edu.sg>.

RAY J. PAUL (Creative Discombobulator) is a Professor of Simulation Modeling, Director of the Centre for Applied Simulation Modeling, creator of the Centre for Living Information Systems Thinking, all at Brunel University, UK. He received a B.Sc. in Mathematics, and an M.Sc. and a $\mathrm{Ph}$.D. in Operational Research from Hull University. He has published widely, in books, journals and conference papers, many in the area of simulation modeling and software development. He has acted as a consultant for a variety of United Kingdom government departments, software companies, and commercial companies in the tobacco and oil industries. He is the editor of the Springer-Verlag Practitioner book series. His research interests are in methods of automating the process of modeling, and the general applicability of such methods and their extensions to the wider arena of information systems. He is currently working on wider aspects of simulation, in particular in WebBased Simulation and the new G2R3 modeling technique. His email and web addresses are <ray.paul@brunel. ac.uk> and <www.brunel.ac.uk/ csstrjp>. Professor Paul has Parkinsonism, but insists on working part-time because he enjoys it.

STEVE REICHENTHAL Steven W. Reichenthal is an Associate Technical Fellow at the Boeing Company. He develops simulations and software applications as a member of the Logistics Engineering organization in Anaheim, California. He has a Masters degree in Computer Science and an MBA, and teaches software development courses at the California State University in Fullerton as an adjunct professor.

STEFFEN STRAßBURGER is head of the department "Virtual Development" at the Fraunhofer Institute for Factory Operation and Automation in Magdeburg, Germany. Previous professional experience includes two years as researcher at the DaimlerChrysler Research Center in Ulm, Germany, where he was involved with research topics in the Digital Factory and Digital Engineering context, especially in the area of simulation integration and distributed simulation. He holds a PhD and a Master's degree in Computer Science from the Otto-von-Guericke University in Magdeburg, Germany. His international experience includes a one-year stay at the University of Wisconsin, Stevens Point and a stay at the Georgia Institute of Technology, Atlanta. He actively participates in several international conferences. His main research interests lie in distributed and web-based simulation, virtual reality, and middleware technologies like the High Level Architecture, CORBA, DCOM, and Web Services. His email address is <strassburger@iff.fraunhofer.de>.

SIMON J. E. TAYLOR is the convener of this panel. He is also Information Director of ACM SIGSIM, ACM SIGSIM PADS Liaison Officer and Chair of the Simulation Study Group of the UK Operational Research Society. $\mathrm{He}$ is a steering committee member of PADS and general co-chair of the UK Simulation Workshop Series. He currently leads the international COTS simulation package interoperability forum (HLA-CSPIF) through SISO (<www . cspif. com $>$ ). He is a Senior Lecturer in the Department of Information Systems and Computing and is a member of the Centre for Applied Simulation Modeling, both at Brunel University, UK. He is also a Visiting Associate Professor at the Parallel and Distributed Computing Centre, Nanyang Technological University (Singapore). He was previously part of the Centre for Parallel Computing at the University of Westminster. He has an undergraduate 
degree in Industrial Studies (Sheffield Hallam), a M.Sc. in Computing Studies (Sheffield Hallam) and a Ph.D. in Parallel and Distributed Simulation (Leeds Metropolitan). His main research interests are distributed simulation and applications of ICT to simulation modeling. He is also a member of the Purple Theatre Company. His email is <simon.taylor@brunel.ac.uk>.

STEPHEN J. TURNER joined Nanyang Technological University (Singapore) in 1999 and is Director of the Parallel and Distributed Computing Centre in the School of Computer Engineering. Previously, he was a Senior Lecturer in Computer Science at Exeter University (UK). He received his MA in Mathematics and Computer Science from Cambridge University (UK) and his MSc and $\mathrm{PhD}$ in Computer Science from Manchester University (UK). His current research interests include: parallel and distributed simulation, distributed virtual environments, grid computing and multi-agent systems. He is steering committee chair and program co-chair of the Workshop on Principles of Advanced and Distributed Simulation and advisory committee member and general chair of the Distributed Simulation and Real Time Applications Symposium. His email address is <ASSJTurner@ntu.edu.sg $>$. 